\title{
The Drug Effectiveness Reporting and Monitoring System: Discussion and Prototype Development
}

\author{
Project Supervisor \\ Dr. Amar Gupta \\ Professor of Entrepreneurship and MIS \\ Thomas R. Brown Chair in Management and Technology \\ Senior Director for Research and Business Development \\ Eller College of Management \\ The University of Arizona \\ Email: gupta@eller.arizona.edu \\ Website: http://next.eller.arizona.edu/ \\ Research Personnel \\ Igor Crk \\ Ph.D. Student in Computer Science \\ University of Arizona \\ Surendra Sarnikar \\ Ph.D. Candidate in MIS \\ Eller College of Management \\ Bipin Karunakaran \\ M.B.A Candidate \\ Eller College of Management
}

University of Arizona

Tucson, AZ 85721 


\section{Abstract}

Medication errors occur commonly in healthcare facilities resulting in adverse drug events. These types of errors and their consequences have been previously observed and studied and it has been shown that simple tracking systems can help to significantly decrease occurrences and consequences of such events. We have found a need for similar monitoring for drugs that have just entered the market and whose potential adverse events are not yet known. Current techniques for post-approval monitoring are not adequate for timely discovery of as-of-yet unknown adverse events. FDA's current approach to identifying drugs with serious adverse effects, the MedWatch system, is inherently slow to identify the culprit drugs and to remove them from the market. Between 1997 and 2005, the system was successful in helping to identify 15 drugs with toxic side-effects. However, on average, it took 5.9 years for the identification phase and the subsequent phase for the removal from of the drug from the market. We propose a system that could help reduce this time by a significant amount. The proposed approach involves a simple on-line surveying system coupled with continuous monitoring by participating pharmacists. We have developed a concept demonstration prototype system and provide recommendations for further work related to the scalability, security, and data consistency aspects of our approach. In addition, we analyze how advances in semantic web and ontology development could be used to populate the database of the proposed system.

Keywords: Adverse event reporting, community pharmacy safety network, post-marketing surveillance, heterogeneous information systems, heterogeneous database systems, healthcare information integration, drug effectiveness reporting, drug effectiveness monitoring 


\section{Introduction}

Timely information on adverse drug effects can save lives and reduce healthcare costs. Previous studies show that more than two million adverse drug reactions occur yearly and result in 100,000 deaths (Lazarou, Pomeranz and Corey, 1998; Gurwitz, Field, Avorn, et al., 2000). In the U.S., the Food and Drug Administration (FDA) is responsible for conducting post-approval monitoring through post marketing surveillance programs that rely, for the most part, on voluntary submission of reports by patients and healthcare providers. The drawbacks of the FDA's approach are poor quality of submitted reports, duplicate reporting of events, underreporting of adverse events capturing only a small fraction of the actual events, and absence of denominator or baseline information required to make meaningful conclusions from the data. We propose a system that features a per-drug customizable baseline and follow-up survey interface, elimination of duplicate reports, and continuous monitoring of patients.

After the introduction of a drug into the market, a bi-directional flow of information related to drug usage, interactions, adverse effects, and treatment outcomes needs to take place between the drug companies, FDA, healthcare professionals and the patients. Timely and effective information flows should occur between the drug companies and FDA, on one side, and between physicians, pharmacists and patients, on the other. This information should incorporate newly detected adverse events, drug interactions, side effects, and recall information. Continuous monitoring is essential (Bates, Cohen, Leape, et al., 2001); further, the presentation of on-line statistical analysis of collected data that may also be presented to pharmacists would facilitate quicker discovery of adverse effects and patient notification. 
Apart from press releases, a limited number of information services and pharmacy databases currently exist, thereby providing some degree of dissemination of information relating to drug interactions and labeling information. The FDA has partnered with more than a hundred organizations to facilitate rapid dissemination of new information on drugs. However, the current information dissemination mechanisms are efficient only in one-way flow of information from FDA and drug companies to physicians, pharmacists and in turn to patients. The process of the reverse flow of information from patients and healthcare practitioners to the FDA on adverse events, medications, and treatment outcomes is weak and confined to MedWatch and other similarly limited mechanisms. There is currently no widely implemented mechanism for the reverse flow of information from patients to the FDA on an on-going basis.

In order to alleviate some of the above problems, we propose a prototype Community Pharmacy Safety Network (CPSN), a novel community-based network of individuals trained to help patients maximize the benefits of their medications and also trained to obtain reliable data for the FDA on the beneficial and adverse outcomes of medicines. This paper describes the information technology aspect of the CPSN project. It summarizes the initial findings of research undertaken to identify the system requirements and presents summary results from preliminary design and prototyping efforts. The following sections describe the MedWatch program, FDA's current mechanism for post marketing surveillance, and summarize its features and shortcomings. Subsequently, the CPSN project is described in terms of its design and features.

In 2000, Barach and Small presented an analysis of non-medical near-miss event reporting systems and identified several factors that are critical in creating a medical event reporting system. The issues of patient privacy, anonymity of participants (including pharmacies and pharmacists), feedback, surveillance, and workload minimization were the critical 
considerations in creating the prototype system. The significance of these critical success factors is taken into consideration while analyzing current and proposed endeavors.

\subsection{MedWatch Program}

The most important source of adverse event information to the FDA is the MedWatch program. In this program, patients and healthcare providers can submit an adverse event report via several mechanisms including an online report form, fax, phone, and mail. The reports are stored in a database, the Adverse Event Reporting System (AERS). The AERS database is made publicly available on the Internet. The AERS database also contains adverse events reported by the drug manufacturers. The adverse events reported are encoded using standard MedDRA terminology. The AERS database is made available to clinical reviewers and researchers on a quarterly basis to detect safety signals and to monitor for drug safety (CPath newsletter, Spring 2005). Similar systems that have been developed and studied with small scale participation show promising results in terms of timely discovery of drug related problems and reduction of serious adverse events (Bates, Teich, Lee, et al, 1999; Evans, Pestotnik, Classen, et al, 1992; Cohen, Kimmel, et al, 2005). A clear benefit of computerized adverse event monitoring is shown by Evans et al (1992). A system, somewhat similar to the one proposed in this paper, is described by Bates et al (1999, 2001, 2005); while meeting requirements differing from those met by our system, the earlier one showed an 80 percent reduction in adverse drug reactions within the short period of time that the system was in use. The study performed by Cohen et al. (2000) showed a reduction in adverse events through an audit of discharged patients and a subsequent nine-month followup. Clearly, the latter system would benefit from the adoption of an on-line approach that encourages continuous participation by pharmacists. 


\subsection{Limitations of MedWatch Program}

Although MedWatch has been successful at identifying critical side effects of drugs, it is inherently slow to respond and is characterized by other major limitations. For the fifteen drugs removed from the market between 1997 and 2005, the system required an average of 5.9 years after market approval to detect and to respond to the toxicity. In addition, the data show that the system has been effective in identifying adverse effects that exhibited themselves relatively early after drug administration, but was unable to find problems that occurred later, during chronic administration, as reported by the US Department of Health and Human Services (1999) as well as Brewer and Colditz (1999). The best example is Vioxx, where increased risk of cardiovascular events (heart attacks and strokes) was only detected during controlled clinical trials (Bombardier, Laine, Reicin, et al 2000).

Another limitation of current event reporting systems is the absence of data on background rates of events and the number of events per number of patients exposed; such data are essential to make a comparative, scientific evaluation of the results. However, due to the voluntary nature of MedWatch reporting, it has become evident that many suspected adverse events are not reported (Ruck, Jennis, Marcus, 2000). In addition, there is a spike in adverse event reports in response to media publicity and concerned letters to healthcare professionals (Danovaro-Holliday, Wood, LeBaron, 2002). The absence of an early alert system that can quantify the relative numbers of adverse events with medicines is a major impediment to the safe use of such medicines.

\subsection{Trends in Information Technology related to Target Application}

Information related to medical drugs is currently held in a number of disparate information systems. As the drug was developed, the relevant data are stored in the computer 
systems of the organization that performed the original drug development work. Additional information is stored in the information systems of the companies that produce the drug. Further, data on clinical trials would be held on other computer systems. By law, a significant amount of the data must be shared with the FDA in the US, and by similar governmental regulatory agencies in other countries. After the drug is approved for sale, it is distributed through a number of channels to the local pharmacies. These pharmacies contain information on who bought the drug, on what date, and in how much quantity. The computers in the offices of doctors and in hospitals are other places that may contain information related to how a particular patient responded to a particular drug.

The issue of integration of information from disparate systems has been an active area of research for a long time as highlighted by Gupta (1989). Techniques for potentially mitigating this problem have been proposed by a number of researchers, including Reddy et al (1994), Redddy et al (1995) and Shaker et al (2002). The growing availability and the use of the Internet led to a concurrent increase in the number of sources of information that could potentially exchange healthcare information resulting in the emergence of new information paradigms such as ones described by Shortliffe (1998), Siau (2003), Teich et al (2002), and Walker et al (2005).

In order to exchange information with efficiency, one needs to ensure that the underlying meaning attached to the information is consistent between the source of the information and the user of the information. For example, if one wanted to find the weather in Paris and did a quick search on the web, one may learn that the temperature is 20 degrees. To a user in the US accustomed to seeing temperatures expressed in Fahrenheit, the immediate perception may be that it is a very cold day in Paris. In reality, the temperature is specified in Centigrade, though this scale of measurement may not be explicitly stated on the concerned website. This is a very 
simple example and can be readily addressed using the concept of mediators, described in Wiederhold and Geneserath (1997). In the case of health care, this mediator technique has been applied by several researchers, including Sarnikar and Gupta (2006). Further, for the healthcare arena, the differences in underlying meanings, or semantics, are more complex in nature, especially in a web environment that encompasses many different countries, cultures, hospitals, and pharmacies. Accordingly, there is a growing emphasis on the issue of semantics, as highlighted in recent papers by Sheth et al (2005), Bry et al (2005), and Priebe (2005). Different fields are characterized by their own sets of vocabularies, or ontologies, and we see newer approaches to the creation and use of ontologies to suit diverse sets of users; see, for example, papers by Kalyanpur et al (2005), Corcho and Gomez-Perez (2005), and Cristani and Cuel (2005).

The healthcare arena is faced by another major constraint. This is the issue of the very high degree of security and privacy that is mandated whenever medical information of individuals is involved. If one surmounted the issues of semantic incompatibility enumerated in the preceding paragraphs, one could design a Drug Effectiveness Reporting and Monitoring System that relied on automatic feed of information from the relevant set of information systems. But, it is very difficult, if not impossible, to secure approvals from multiple Internal Review Boards (IRBs) to such release of information. So, it was decided to build a concept development prototype system that was primarily autonomous in nature. At a future time, when the Food and Drug Administration, and other cognizant regulatory agencies, frame new guidelines for sharing of information in situations where human lives are endangered, one could consider using the newer techniques to populate the database of the prototype system described in the following sections of this paper. 


\section{CPSN System}

One of the key facets of the CPSN project is to build a large-scale data collection mechanism that would involve pharmacists and pharmacy technicians in identifying and collecting adverse event information. For outpatient prescription drug therapy, pharmacists and pharmacy technicians are usually the first point of contact with patients. With proper infrastructure and incentives, these pharmacists and pharmacy technicians could potentially maintain comprehensive historical information on the patient's medication history detailing the different types of medications taken by the patient and the duration for which each medication has been taken. Such records can serve as an alternative source of information for evaluating the longterm effects of clinical medicines.

Current pharmacy information systems are mostly focused on supporting billing and electronic prescription workflow management. In order to collect information that would be more useful for pharmaco-epidemiological purposes, a plan for a new web-based information system was developed. This system can be integrated with pharmacy information systems, and can enable collection, analysis and timely dissemination of adverse and beneficial drug effectiveness information. The key system features are described in the following subsections of this paper.

\subsection{Overall Architecture}

From the beginning, we envisaged that the data collection mechanism should be entirely webbased and should utilize ordinary web browsers for access, minimizing the need to install specialized software on heterogeneous platforms. The benefits and drawbacks of this approach 
have previously been analyzed in (Luxenberg, DuBois, Fraley, et al, 1997). We feel that at this time, the drawbacks of security problems described by Luxenberg et al. (1997) have largely been overcome. The proposed architecture will also reduce the overall cost of the system, and facilitate easier maintenance and updates. A majority of surveyed pharmacies maintain an internet access mechanism; hence access to the proposed system should be feasible from a large number of places. When the system is scaled to a national level, a large amount of data will become available; the availability of such information will provide greater ability to detect rare events earlier. Considering security and scalability issues, either a centralized database cluster or a federated database server integrating multiple distributed databases could be used. For the present, we have adopted the former option. As discussed in section 1.3, the latter approach could be utilized at a later date.

\subsection{System Components}

The CPSN Web system, shown in Figure 1, is comprised of three major modules: an information collection and follow-up module; surveillance and monitoring module; and a surveillance administration module.

2.2.1 Information Collection Module: The information collection module incorporates the data collection process. The functionality of this module is primarily of relevance to pharmacy technicians and pharmacists. Figure 2 describes the interrelationships among the collection of baseline and follow-up data regarding drug usage and effects. This module facilitates the drug specific data collection processes by bringing up appropriate questionnaires and data collection forms, and also generates follow up triggers and alerts based on pre-specified rules 


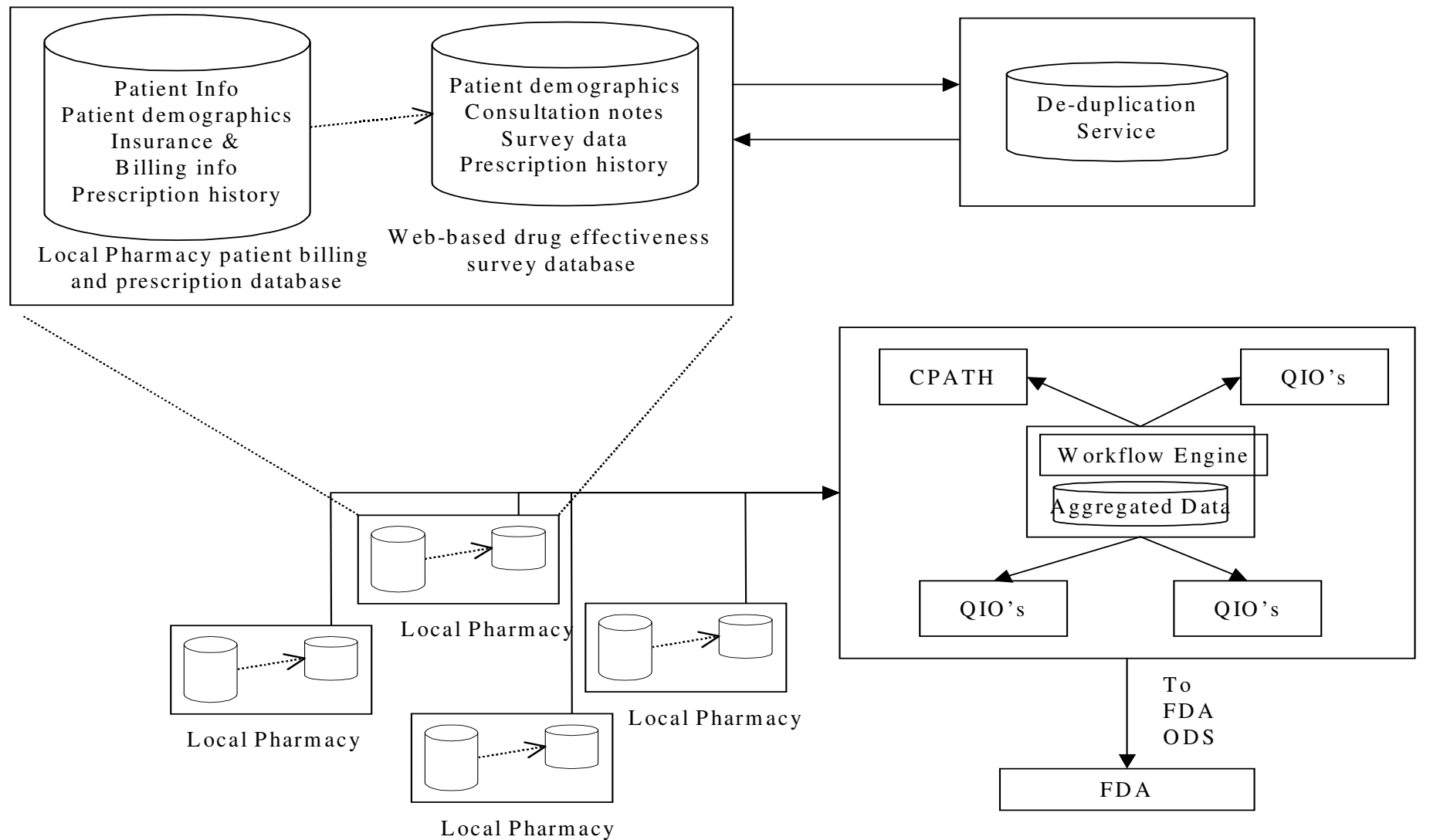

Local Pharmacy

Figure 1. Overall Architecture 


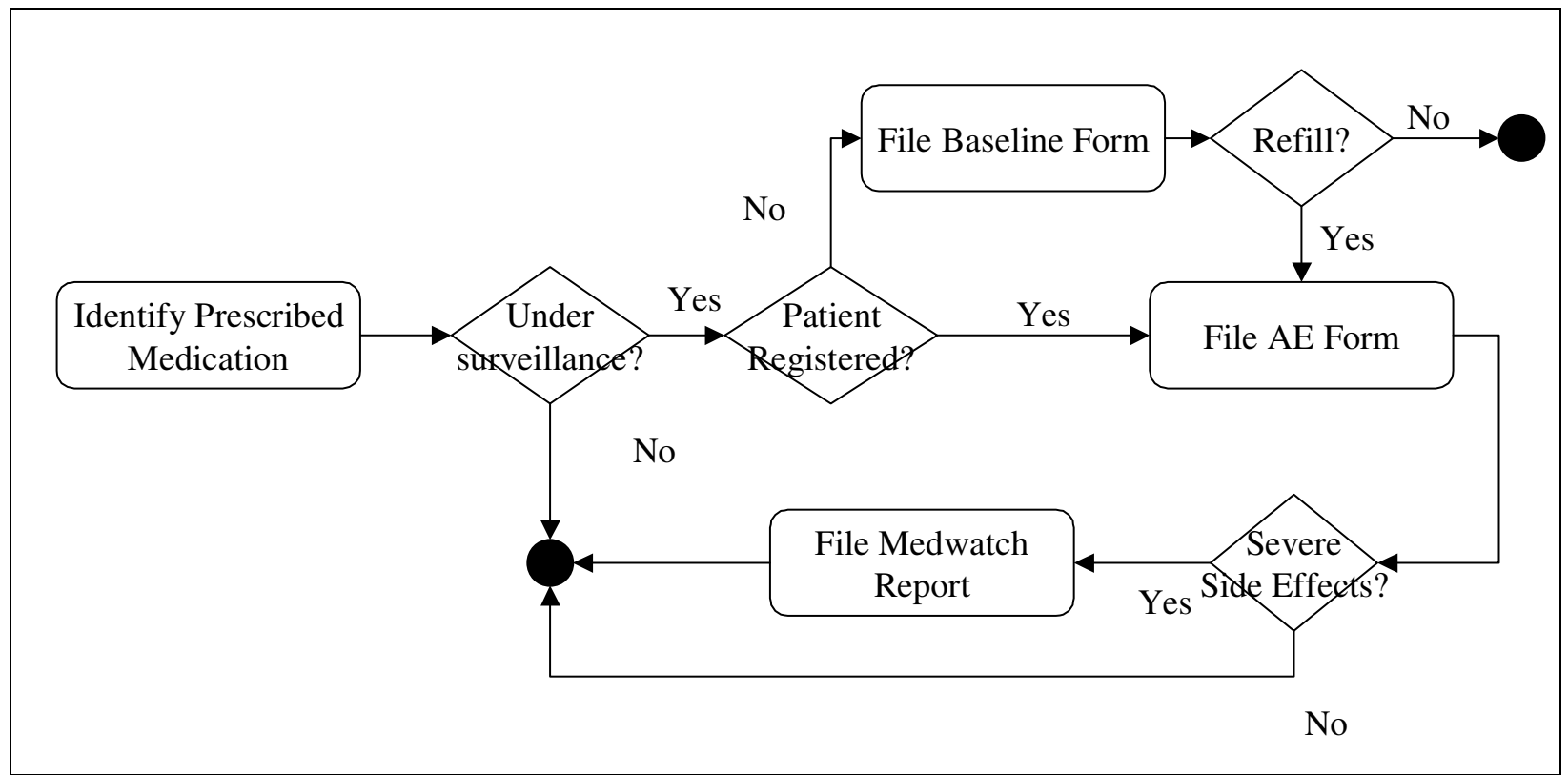

Figure 2. Data Collection Process

This module facilitates the drug specific data collection processes by bringing up appropriate questionnaires and data collection forms, and also generates follow up triggers and alerts based on pre-specified rules.

2.2.2 Surveillance and Monitoring Module: The surveillance and monitoring module presents summary information on the data that have been collected. The functionality of the surveillance module helps researchers and pharmaco-epidemiologists in signal identification and hypothesis generation. The module incorporates visualization tools that support the use of relational queries for trend analysis and summary data visualization. For more complex statistical analysis and data mining applications, this module provides a download interface for integrating data with the commonly used analytics software such as Qscan, Spotfire, and SPSS. 


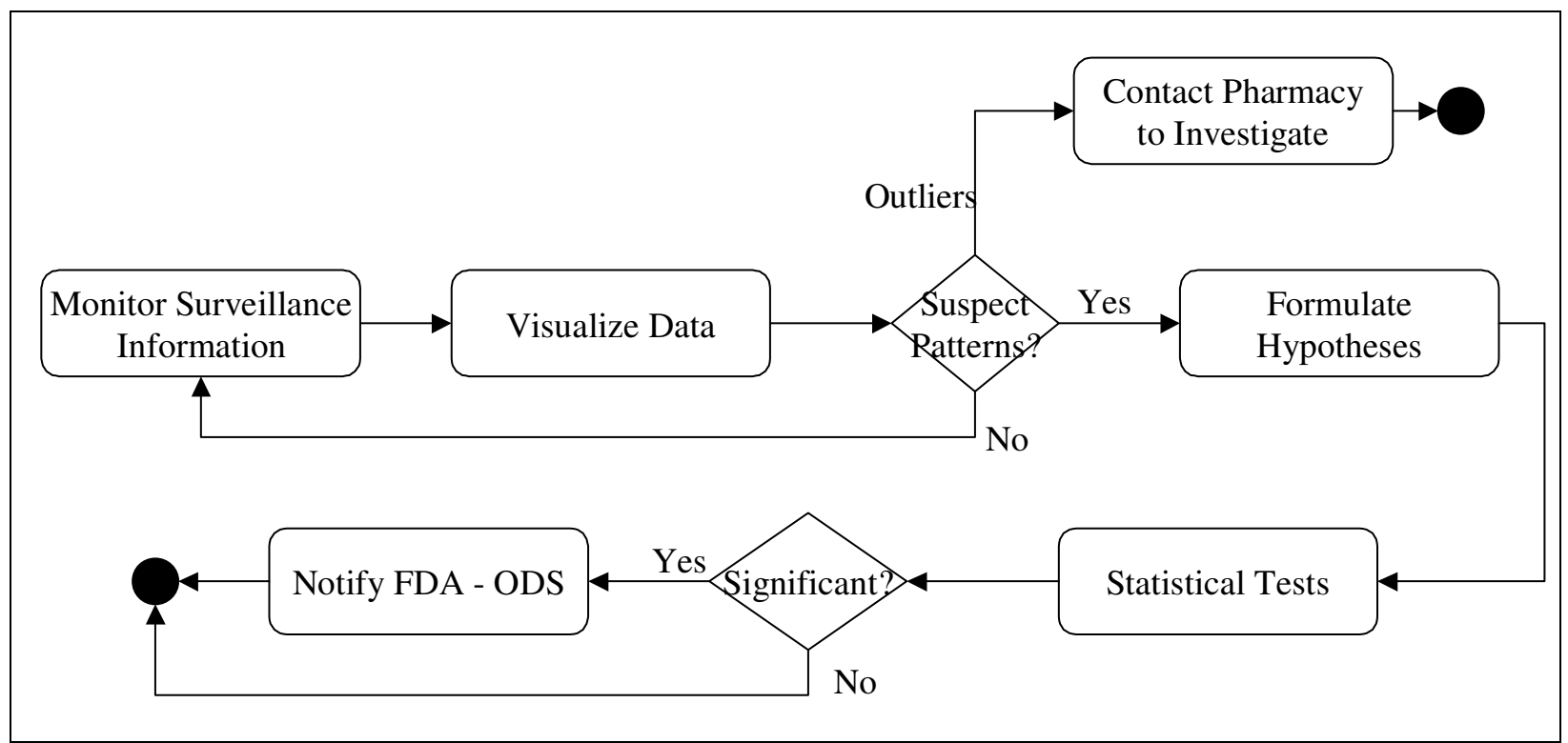

Figure 3. Surveillance Process

2.2.3 Surveillance Administration Module: The surveillance administration module enables the initiation of the surveillance process for new drugs. This module incorporates algorithms for the creation of follow up questionnaires and for setting up custom triggers and schedules. Since the type of information collected varies by the type of the drug, the module enables creation of customized questionnaires by selecting a set of questions from a database or by creating new ones. Once the surveillance process is initiated, participating pharmacies can be notified of the update and can receive instructions on the process.

2.2.4 Data Security and Privacy: The proposed system must ensure that the privacy rights of the patients are adequately protected and that the collected data are protected from unauthorized access. Standard security measures such as the use of 128-bit Secure Sockets Layer (SSL) for secure transmission and database encryption and the use of firewalls for security of stored data can be implemented into the CPSN system to provide the desired data security. In order to protect patient privacy rights, HIPAA regulations need to be complied with. Note that HIPAA regulations permit the disclosure of protected health information (PHI) without authorization for adverse event reporting. The patients are primarily identified in the system by a consumer id, the 
first name and the last name. The first name and the last name of the patient are stored in the database in an encrypted form and can be viewed only by authorized scientists. A patient can be identified only by linking the consumer id and the patient name in the system database with corresponding information in the local pharmacy's database. Since this access and privilege is available only to the pharmacist, the patient privacy is adequately protected. Adequate privacy at all levels of the system can be attained by the use of a cryptographic hashing function with low collision probability, performed on identifying patient information, such as name and social security number. The use of such an approach would adequately protect the identity of the patient when such information is stored at an off-site database while at the same time ensuring the uniqueness of records for each patient and significantly reducing the possibility of duplicate records. Further, the use of cryptographic techniques with semantic annotation for localization and access of patient records across institutional boundaries has been suggested by Eichelberg, Aden, and Thoben (2005).

2.2.5 Duplicate Record Identification: Although the current system provides better safeguards against duplicate records than the current the MedWatch system, duplicate records can still be introduced into the database due to a number of factors such as the use of local identifiers (in place of social security numbers), the migration of patients between participating pharmacies and the presence of duplicates in the pharmacy information systems itself. A probabilistic mechanism will need to be implemented in order to identify and merge duplicate records. Based on the similarity of names, age, gender, current and previous medications and dosages, and details of allergies, duplicate records can be identified with a certain confidence level. This confidence level can be used as the basis for automated elimination of duplicate records during the analysis phase and also as a tool for manual elimination of duplicated records by a data cleaning expert. 
The use of information filters has been examined by a number of researchers, including Sarnikar,et al (2005).

2.2.6 Information Collection and Repository: The purpose of the overall system is to collect information that is needed for drug safety and pharmaco-epidemiological analysis. Based on a preliminary analysis of some of FDA's post marketing surveillance programs and the associated research and monitoring process, in conjunction with preliminary questionnaires designed by the Center for Educational Research Therapeutics, the University of Arizona project team developed five types of questionnaires and forms: basic patient information form; baseline information questionnaire; routine follow-up questionnaire; special follow-up questionnaire (for liver disease, reason for discontinuation); and adverse event reporting form. The patient information form is used to collect information on patient demographics such as age, ethnicity, and gender, as well as background patient information such as diabetes and other chronic conditions. The baseline information questionnaire is used to collect information on the patient's health status before the start of a medication. The routine follow up questionnaire is utilized to record the patient's health status at every refill of the medication. Some medications are known to have potential harmful side effects usually occurring after a certain period of time has elapsed. In order to capture this type of information, special follow up questionnaires can be designed at the time of initiation of surveillance. At such a stage, one can also create a schedule for the administration of customized questionnaires. The system is designed to prompt the pharmacist at the scheduled times (for example: eight weeks after start of medication for liver diseases) to contact the patient to query the latter for specific symptoms. Special follow-up questionnaires are also used to elicit relevant information from patients who have opted to discontinue seeking refills of prescription drugs. 


\section{Prototyping Effort}

In order to identify potential issues that could arise during actual system implementation, a proof-of concept prototype system was developed using PHP and MySQL database on a Linux platform. The prototyping efforts were geared towards obtaining a better understanding of the requirements and to predict potential technical and usability issues that may arise while implementing and deploying a production system on a local basis, a state-wide basis, or a nationwide basis. The prototype can be accessed at the following URL:

http://seurat.ecom.arizona.edu/ derms/phpdraft/.

\subsection{System Scalability}

The functional components of the prototype system were coded in PHP. The system is hosted with Apache, and the backend database is supported by the MySQL database system. PHP, Hypertext Preprocessor, is a reflective programming language used for developing server-side applications and dynamic web content. It was chosen for its versatility, ease of interfacing with a database system (such as MySQL), and potential for creating a highly scalable system. Since the processing of PHP pages is done server-side, the scalability of the final application depends solely on the hardware infrastructure that is hosting the application. PHP also allows for a modular application design that calls for components of that application to operate independently of other components. As such, the process of addition of features and new components is made relatively safe and easy. PHP is an alternative to ASP.NET, ColdFusion, JSP/Java, and CGI/Perl systems. Its use in large-scale systems such as phpBB and MediaWiki is a testament to its popularity and speaks of the versatility of its uses.

The MySQL database system is a multithreaded, multi-user, SQL database management system. Since the introduction of MySQL Cluster, a technology that adds shared-nothing 
clustering capabilities to MySQL, MySQL offers the capacity for high-availability, highperformance, and near-linear scalability.

\subsection{Infrastructure Requirements}

Ideally, the infrastructure hosting the final system would be dedicated solely to hosting the system. The performance of benchmark tests on such a setup would provide accurate estimates of the capability of the system to meet the daily demands that would be placed on the system by pharmacy traffic and database manipulation operations. The infrastructure described here can be potentially scaled up to meet the bandwidth requirements for the deployment of such a system on a national and international basis.

The prototype infrastructure would include a dedicated web-server with PHP support, coupled with a dedicated MySQL server. This architecture could potentially address the demands of hundreds of concurrent users, based on the low bandwidth requirements per user and the simple interfaces developed for the existing prototype. Anticipating an increase in the demand on the infrastructure, the single web-server could be expanded into a web-server cluster. In such a setup, a single master node would route traffic to a server that is experiencing a lower load than the others. This algorithm would ensure load balancing across the cluster and would also enhance availability, since the cluster will continue to provide its services as long as at least the master node and a client node can communicate with each other.

Similarly, a MySQL cluster would be able to meet the demands of a growing dataset and traffic load even in an environment involving hundreds of pharmacists. This clustering system was designed for high availability, performance, and reliability. However, the scaling-up of the system to cater to the needs of pharmacists spread across many states would need to be explored in greater detail. 


\subsection{System Benefits}

Apart from providing an easy and comprehensive acquisition of information on drug effectiveness information, the proposed system can greatly contribute towards the creation of a rapid response comprehensive post marketing surveillance system. The perceived direct and indirect benefits are as follows:

3.3.1 Creation of Longitudinal Medical Records: The aggregation of baseline medical information and periodically collected follow-up medical information lead to comprehensive longitudinal medical records. This integrated medical record can enable better identification of beneficial effects of drugs and hard to detect adverse events that are characterized by long manifestation periods.

3.3.2 Faster detection of adverse events: Since the monitoring is systematic and takes place at the point of medication dispensation, the coverage of adverse events is expected to be wider and more accurate, enabling faster detection of adverse events.

3.3.3 Reduced time to market for new and critical drugs: Phase 3 trials represent the most expensive and time consuming phase in the drug approval process. Despite their rigor, some adverse effects remain undetected during the clinical trial processes. The longer the time it takes to detect the adverse events, the bigger is the need for more comprehensive, expensive and longer duration phase 3 trials. A fast detection system reduces these risks, and can make it possible to speed up time to market for new drugs. This accelerated time to market is especially crucial in cases where a promising drug could have a beneficial impact on large sections of populations such as drugs for AIDS.

3.3.4 A step towards open research: Naeve (2005) characterizes the current state of medical research is that of closed research, representing a gap between research and practice. This 
is anecdotally evidenced in the relatively long adverse effect detection time seen with the systems currently in place. The dataset that would be made available by the proposed system bridges the gap of information flow between patients and researchers, allowing for more timely adverse effect discovery as well as facilitating research of drug interactions. The implementation of the proposed system and related policies shows promise as a step towards reducing entropy in the drug effectiveness monitoring systems, with implications for both furthering research and protecting consumers. As such, it fits squarely in the application category of Semantic Web and Information Systems, as described by Lytras (2005).

\subsection{Limitations}

The proposed drug effectiveness monitoring and adverse event reporting system is more comprehensive than the current MedWatch system. However, it suffers from the following limitations:

3.4.1 Inability to track drug samples: Most new drugs are initially handed out as samples to patients. The prototype system will be able to capture information only when the patient uses a pharmacy to fill or refill the prescription. However, no mechanism has been devised yet to track this information when sample drugs provided by the physician cover all or part of the duration of medication and the patient does not contact or visit the pharmacy.

3.4.2 Limited tracking for comparative effectiveness: The system is currently designed to collect data only for certain drugs selected for surveillance. As such, there are currently limited evidence gathering methods focusing on comparative effectiveness of different drugs treating a particular condition. 
3.4.3 Time constraints and shortage of pharmacists: Due to the pharmacists' time constraints and a shortage of pharmacists, the deployment of the described system may be constrained by the pharmacists' workload. However, financial incentives can promote the adoption of the proposed system, as suggested by Farris, Kumbera, Halterman, and Fang (2002).

3.4.4 Duplicate records: The current system design partly relies on the consumer id assigned by the community pharmacy to the patient to uniquely identify him or her. This system is helpful in tracking patients when they use the same or different stores of the same pharmacy chain and when the pilot study is confined to a single pharmacy chain. However, the heterogeneity of information technology in pharmacies, as shown in Table 1 , makes the elimination of duplicate records a critical factor in the success of a widescale adoption of the proposed system. Eichelberg, Aden, and Thoben (2005) suggest a protocol that combines cryptographic techniques with semantic annotation for localization and access of patient records across institutional boundaries.

3.4.5 Privacy Regulations: Apart from compliance with HIPAA regulations, the system will need to comply with the privacy policies of the participating pharmacies. The privacy policy of most pharmacy companies require that Protected Health Information would be disclosed to researchers only if an institutional review board has approved the research proposal and established protocols have been incorporated to ensure the privacy of the information.

\section{Operating Environment and Recommendations}

The privacy related policies of the key pharmacy chains in Arizona are summarized in Table 1. 


\begin{tabular}{|c|c|c|c|c|}
\hline Pharmacy & $\begin{array}{l}\text { Number } \\
\text { of stores in } \\
\mathrm{AZ}\end{array}$ & $\begin{array}{l}\text { Pharmacy } \\
\text { Information system }\end{array}$ & Online facilities & Privacy policy \\
\hline Wal-mart & 52 & $\begin{array}{l}\text { In-house } \\
\text { (Windows based) }\end{array}$ & $\begin{array}{l}\text { Online refilling, } \\
\text { Online } \\
\text { refill history, } \\
\text { Prescription } \\
\text { transfers }\end{array}$ & $\begin{array}{l}\text { Discloses } \\
\text { information for } \\
\text { authorized } \\
\text { research }\end{array}$ \\
\hline Walgreens & $>120$ & $\begin{array}{l}\text { Intercom plus } \\
\text { (Windows based) }\end{array}$ & Prescription refills & $\begin{array}{l}\text { Discloses } \\
\text { information for } \\
\text { quality } \\
\text { assurance } \\
\text { activities and } \\
\text { for authorized } \\
\text { research }\end{array}$ \\
\hline Safeway & $\approx 60$ & PDX & $\begin{array}{l}\text { Refill, transfer \& } \\
\text { delivery }\end{array}$ & Not available \\
\hline Fry's Food & 108 & In-house & Refill & $\begin{array}{l}\text { Discloses } \\
\text { information for } \\
\text { authorized } \\
\text { research }\end{array}$ \\
\hline Osco Drug & $>100$ & No Information & $\begin{array}{l}\text { Refill, transfer \& } \\
\text { delivery }\end{array}$ & $\begin{array}{l}\text { Discloses } \\
\text { information for } \\
\text { authorized } \\
\text { research }\end{array}$ \\
\hline $\begin{array}{c}\text { Bashas' } \\
\text { Supermarkets }\end{array}$ & 35 & QS1 & $\begin{array}{l}\text { Prescription } \\
\text { Transfer, } \\
\text { Prescription } \\
\text { compounding }\end{array}$ & $\begin{array}{l}\text { Discloses } \\
\text { information for } \\
\text { authorized } \\
\text { research }\end{array}$ \\
\hline CVS/Pharmacy & $\begin{array}{l}\text { No } \\
\text { information }\end{array}$ & In-house developed & $\begin{array}{l}\text { Prescription orders } \\
\& \text { refills }\end{array}$ & $\begin{array}{l}\text { Discloses } \\
\text { information for } \\
\text { authorized } \\
\text { research }\end{array}$ \\
\hline
\end{tabular}

Table 1. Policies of Pharmacies in Arizona 
Walgreens, which generates the highest volume of sales, has stores linked by satellite system that allows prescriptions to be refilled at any retail site. This was accomplished with its “Intercomp" computer system, Walgreens' pharmacy computer and workflow system (Windows based) is claimed to allow pharmacists to spend more time counseling patients by assigning administrative tasks to pharmacy technicians. The privacy notice of Walgreens has a specific clause stating that patient information could be used in an effort to continually improve the quality and effectiveness of the health care and service provided.

Walmart claims to have perfected low cost distribution in the pharmacy area. The world's largest retailer is also the fourth largest pharmacy retailer and has a windows based integrated information system.

Safeway utilizes the PDX pharmacy computer system and practices a workflow model that was designed to allow maximum contact with patients. Safeway maintains an online facility that allows prescriptions to be refilled, transferred and delivered. It also provides a drug interaction tool for learning how medicines interact with other medicines, supplements, and foods.

\section{1 Randomized Control Trials for Pharmaceutical Care Program}

Between 1997 and 1999, randomized control trials were conducted to study the effectiveness of pharmaceutical care programs in Indianapolis. The pharmaceutical care program was aimed at giving the pharmacists a greater role in providing the patients with better healthcare. We are referring to this study as we find that the observations and conclusions are very relevant to our project.

The study was initiated with Revco pharmacy, which was subsequently taken over by CVS as the controlled trials began. Originally, patient data from Revco was being transferred to 
INPC (Indianapolis network for patient care) for purposes of the study. But after the takeover, due to the changes in the computer systems and more due to the changes in the data sharing policies, the researchers had to rethink their strategies to a large extent. The main hurdle was that CVS prohibited transfer of patient data to INPC without patients explicitly signing "opt-in" forms.

After discussions with the researchers, CVS mailed the opt-in forms to the identified pool of consumers who were potential participants in the study, apprising them of the evaluation process and promising $\$ 60$ to each of them for their participation in the study. Of the persons to whom such forms were mailed, $21 \%$ responded, with $15 \%$ expressing willingness and $6 \%$ refusing consent. CVS representatives tried to reach individuals who did not respond and were able to contact less than half of them, Finally, they had obtained explicit consent from roughly $20 \%$ of the persons that had been identified initially. These consumers were interviewed and after ensuring their eligibility, their names were transferred to the project team.

At the time of the above study, it was not technologically possible to integrate the research programs with the CVS store computers. But advances in network architecture standards now enable such integrations. In fact, CVS is one of the first drug retailers to integrate the Internet and traditional store services and today maintain an extensive database of purchases made by customers.

\subsection{Recommendations}

The above experience of Revco/CVS shows that consumers are generally reluctant to provide explicit approval for use of their records on medications, even when financial incentives are provided and the intent of the use is for study purposes. 
Based on the experiences during the study, the research team came up with the following recommendations:

- Involvement of pharmacists: The success of the proposed Drug Effectiveness Reporting and Monitoring System is heavily dependent on support and compliance on the part of the pharmacists. While financial incentives to them may be helpful, the program can go ahead only if they can be motivated, even mandated, to consider entering information into the proposed system to be an integral part of their job. One needs to still decide whether one pharmacist should be designated in each large pharmacy to do this job, or whether it should made the collective responsibility of all pharmacists who work in large pharmacies.

- Securing corporate buy-in: Since the research would involve large pharmacy chains that are corporations, aligning the research objectives to the long-term goals of these corporations will be desirable. This would involve interactions with persons at various decision-making levels in these corporations.

- Ensuring compliance to pharmacy privacy policy guidelines: The privacy policies of retail pharmacies are different from one another, and these differences need to be taken into account while finalizing the strategy, especially if the concept of semantic web is used at a later date to populate the database of the proposed system.

- Data mining and knowledge discovery: The current concept demonstration prototype system focused primarily on the collection of data on adverse effects from patients. The capabilities of such systems can be greatly enhanced through the use of emerging data mining and neural network approaches. 


\section{Conclusion}

In this document, we have described the features of the drug effectiveness reporting and safety monitoring system and its usefulness in post marketing surveillance in an operating environment that is characterized by considerable diversity in terms of the underlying semantics of the key pieces of information. Future work would involve time and motion studies in pharmacies to study the work processes of pharmacists and pharmacy technicians. Such studies will lead to the development of usability requirements for the proposed system and to additions and improvements to the current system design and features described in this paper. Parallel research on the semantic web and the creation of ontologies offers the potential for use of automated techniques for populating the proposed Drug Effectiveness Reporting and Monitoring system.

\section{Acknowledgement}

The authors thank Dr. Raymond L. Woosley and others of The Critical Path Institute for their sponsorship of this project involving research and development of the concept development prototype system.

\section{References}

1. Center for Drug Evaluation and Research (n.d.). Adverse Event Reporting System. Retrieved from: http://www.fda.gov/cder/aers/default.htm

2. C-Path Newsletter (Spring 2005).

3. Barach, P. and Small, S. (2000). Reporting and preventing medical mishaps: lessons from non-medical near miss reporting systems. British Medical Journal, 320,759-763. 
4. Bates, D.W., Cohen, M., Leape, L.L., et al. (2001). Reducing the frequency of errors in medicine using information technology. Journal of the American Medical Informatics Association. 8, 299-308.

5. Bates, D.W., Teich, J.M., Lee, J., Seger, D., Kuperman, G.J., Ma'luf, N., Boyle, D., Leape, L. (July, 1999). The impact of computerized physician order entry on medication error prevention. Journal of the American Medical Informatics Association, 6(4), 313-321.

6. Bates, D.W., Evans, R.S., Murff, H., Stetson, P.D., Pizziferri, L., Hripcsak, G., (2003). Detecting adverse events using information technology. Journal of American Medical Informatics Association, 10, 115-28

7. Bombardier, C., Laine, L., Reicin, A., et al (2000). Comparison of Upper Gastrointestinal Toxicity of Rofecoxib and Naproxen in Patients with Rheumatoid Arthritis. The New England Journal of Medicine, Vol. 343, 1520-1528.

8. Brewer, T., and Colditz, G.A., (1999). Postmarketing Surveillance and Adverse Drug Reactions: Current Perspectives and Future Needs. Journal of the American Medical Association, 281, 824-829.

9. Bry, F., Koch, C., Furche, T., Schaffert, S., Badea, L. \& Berger, S., (2005). Querying the Web Reconsidered: Design Principles for Versatile Web Query Languages. International Journal of Semantic Web and Information Systems. Vol. 1, Issue 2, 1-21.

10. Cohen, M.M., Kimmel, N.L., et al. (2005). Medication Safety Program Reduces Adverse Drug Events in a Community Hospital. Quality and Safety in Health Care, 14, 169-174.

11. Corcho, O. \& Gomez-Perez, A., (2005). A Layered Model for Building Ontology Translation Systems. International Journal of Semantic Web and Information Systems. Vol. 1, Issue 2, $22-48$.

12. Cristani, M., Cuel, R. (2005). A Survey on Ontology Creation Methodologies. International Journal of Semantic Web and Information Systems. Vol. 1, Issue 2, 49-69. 
13. Danovaro-Holliday, M.C., Wood, A.L., LeBaron, C.W., (2002). Rotavirus Vaccine and the News Media. Journal of the American Medical Association, 287, 1455-1462.

14. Eichelberg, M., Aden, T., Thoben, W. (2005). A distributed Patient Identification Protocol Based on Control Numbers with Semantic Annotation. International Journal on Semantic Web and Information Systems, Vol. 1, No. 4, 24-43.

15. Evans, R., Pestotnik, S., Classen, D. et al. (1992). Prevention of adverse drug events through computerized surveillance. Proceedings of the Annual Symposium of Computer Applications in Medical Care, 437-41.

16. Farris, KB, Kumbera, P., Halterman, T. and Fang, G (2002). Outcomes-based Pharmacist Reimbursement: Reimbursing Pharmacists for Cognitive Services. Journal of Managed Care Pharmacy, (8)5, 383-93

17. Gupta, A. (ed.) (1989). Integration of Information Systems: Bridging Heterogeneous Databases. IEEE Press.

18. Gurwitz, J.H., Field, T.S., Avorn, J. et al. (2000). Incidence and preventability of adverse drug events in nursing homes. American Journal of Medicine, 109, 87-94.

19. Kalyanpur, A., Parsia, B., Hendler, J. (2005), A Tool for Working with Web Ontologies. International Journal of Semantic Web and Information Systems. Vol. 1, Issue 1, pp:36-49

20. Lazarou, J., Pomeranz, B., Corey, P. (1998). Incidence of Adverse Drug Reactions in Hospitalized Patients. Journal of the American Medical Association, 279, 1200-1205.

21. Luxenberg, S.N., DuBois, D.D., Fraley, C.G., et al. (1997). Electronic forms: benefits and drawbacks of a World Wide Web-based approach to data entry. Proceedings of the AMIA Annual Fall Symposium., 804-8. 
22. Lytras M., (2005), Semantic Web and Information Systems: An Agenda Based on Discourse with Community Leaders. International Journal of Semantic Web and Information Systems, Vol. 1, Issue 1, pp:i-xii

23. Naeve, A., (2005), The Human Semantic Web Shifting from Knowledge Push to Knowledge Pull. International Journal of Semantic Web and Information Systems. Vol. 1, Issue 3, 1-30.

24. Reddy, M. P., Prasad, B. E., Gupta, A., (1995). Formulating Global Integrity Constraints During Derivation of Global Schema. Data and Knowledge Engineering. Vol. 16, 241-268.

25. Reddy, M. P., Prasad, B. E., Reddy, P. G., Gupta, A., (1994). A Methodology for Integration of Heterogeneous Databases. IEEE Transactions on Knowledge and Data Engineering. Vol. 6, No. 6 .

26. Ruck, B., Jennis, T., Marcus, S. (2000). Few NACCT Abstracts of Adverse Drug Reactions are Reported to MEDWATCH. Internet Journal of Medical Toxicology, 3(2), 4. Retrieved from: http://www.ijmt.net/ijmt/3_2/3_2_4.html.

27. Sarnikar, S. and Gupta, A., (2006). A context-specific mediating schema approach for information exchange between heterogeneous hospital systems. International Journal Healthcare Technology and Management. Forthcoming.

28. Sarnikar, S., Zhao, J. L. and Gupta, A. (2005). Medical Information Filtering Using Contentbased and Rule-based Profiles. Proceedings of the Americas Conference on Information Systems.

29. Shaker, R., Mork, P., Barclay, M., Tarczy-Hornoch, P., (2002). A rule driven bi-directional translation system for remapping queries and result sets between a mediated schema and heterogeneous data sources. Proceedings of the AMIA Symposium, 692-696 
30. Sheth, A., Ramakrishnan, C., \& Thomas, C., (2005), Semantics for the Semantic Web: The Implicit, the Formal and the Powerful. International Journal of Semantic Web and Information Systems, Vol. 1, Issue 1, 1-18.

31. Shortliffe, E.H., (1998). The evolution of health-care records in the era of the Internet, Medinfo 9(suppl), 8-14.

32. Siau, K., (2003). Health Care Informatics. IEEE Transactions on Information Technology in Biomedicine, 7(1).

33. Teich, J., Wagner, M., Mackenzie, C., Schafer, K., (2002). The informatics response in disaster, terrorism, and war. Journal of American Medical Informatics Association. 9(2), 97104.

34. T, Priebe T, (2005). A Context-Based Approach for Supporting Knowledge Work with Semantic Portals. International Journal of Semantic Web and Information Systems. Vol. 1, Issue $3,64-88$.

35. US Department of Health and Human Services (May, 1999). Managing the Risk from Medical Product Use, Creating a Risk Management Framework. Taskforce on Risk Management Report.

36. Walker, J., Pan, E., Johnston, D., Adler-Milstein, J., Bates, D. W., Middleton, B., (2005). The Value Of Health Care Information Exchange And Interoperability. Health Affairs.

37. Wiederhold, G., Genesereth, M., (September, 1997). The Conceptual Basis for Mediation Services. IEEE, 38-47. 


\section{About the Authors}

Dr. Amar Gupta is the Tom Brown Endowed Chair of Management and Technology at the University of Arizona in Tucson. Earlier, he held various positions at MIT over a period of 25 years (1979-2004). He co-supervised a major initiative, funded by DARPA, on intelligent integration of information. He has served as an advisor to the World Health Organization in the area of medical informatics, and is funded by NIH for research on developing new agile healthcare information systems for use in emergencies. His project teams have developed new techniques for reading mammograms, and for drug effectiveness reporting and monitoring. Igor Crk is a Doctoral Student at the Department of Computer Science, University of Arizona. He has a B.A. in Computer Science and Mathematics from Carthage College and a M.S. in Computer Science from the University of Arizona. His current research interests are in operating systems, networks, machine learning, and energy management.

Surendra Sarnikar is a Doctoral Student in MIS at the University of Arizona. He has an undergraduate degree in Engineering and a Masters in Management Information Systems. His research interests are in the area of healthcare data and communication standards, medical information filtering, and medical event reporting systems. He has also presented papers on medical information filtering and information distribution mechanisms at the Americas Conference on Information Systems.

Bipin Karunakaran is a graduate dual-degree student for the Master of Business Administration and Master of Science in Management Information Systems program at the Eller College of Management, University of Arizona. He has an undergraduate degree in Electronics engineering and over 10 years of work experience in industrial process automation. His areas of interest are technology strategy and enterprise application integration. 